\title{
Research on the Employment Situation of Chinese College Students in the Prevention and Control of Major Public Health Events
}

\author{
Hairong Ling1,2, Jun $\mathrm{Li}^{2}$, Jing Wang1, Shi Chen ${ }^{*}$ \\ ${ }^{1}$ School of Education Science, Nanjing Normal University, Nanjing, China \\ ${ }^{2}$ Nanjing Communication College, Nanjing, China \\ Email: *180601021@njnu.edu.cn
}

How to cite this paper: Ling, H. R., Li, J., Wang, J., \& Chen, S. (2021). Research on the Employment Situation of Chinese College Students in the Prevention and Control of Major Public Health Events. Psychology, 12, 1230-1245.

https://doi.org/10.4236/psych.2021.128077

Received: July 12, 2021

Accepted: August 10, 2021

Published: August 13, 2021

Copyright $\odot 2021$ by author(s) and Scientific Research Publishing Inc. This work is licensed under the Creative Commons Attribution International License (CC BY 4.0).

http://creativecommons.org/licenses/by/4.0/

\begin{abstract}
The current outbreak of Covid-19 (coronavirus disease of 2019) affected Chinese college students graduating and studying in 2020. The purpose of this article is to study the impact of Covid-19 and analyze ways to control the career adaptability among college students' employment mentality and emotion. A total of 1229 valid questionnaires from participants in three universities in Jiangsu province were collected and analyzed using descriptive statistics and correlation analysis. The study found that: 1) Because of the epidemic, there has been a delay in the reopening of schools, which has caused emotional disturbance in students as their studies and, in turn, their employment are adversely affected. 2) The epidemic has resulted in both "risks" and "opportunities." 3) During the epidemic, students have chosen different ways to apply for jobs, which results in different influences. 4) In emergency situation, the importance of career adaptability is obvious. During the outbreak of epidemic crisis, colleges should guide the students to develop ideology quickly and help them turn "risks" to "opportunities." Besides, colleges should build and actively participate in the "internet field" based on the social linkage mechanism. At the same time, colleges need to build a developing career education mechanism of "change" and "change-coping," which promotes the adaptability and the development of students, thus helping them cope with shifting events by sticking to a fundamental principle.
\end{abstract}

\section{Keywords}

Epidemic, Employment Mentality, Emotion, Employment Information Channel, Career Adaptability 


\section{Introduction}

In China, the outbreak of Covid-19 has had a great impact on the learning as well as the employment situation of college students, and it has affected the employment status, employment channels, and employment psychology of the university students. It is found that the students can experience strong anxiety under random uncertain stimulation (Grillon et al., 2004); students with high anxiety are more likely to be indecisive in career decision-making (Zhang et al., 2018). Under this epidemic situation, the Chinese college students' employment status and their psychological state related to it are worthy of attention and research. Moreover, the sudden epidemic has forced the method of recruitment to change from the previous offline recruitment mode, supplemented by online services, to the online mode, namely, the "cloud recruitment," which is both an innovation and a challenge to many aspiring students. How to participate in the cloud-based recruitment, what role does "cloud" play in the recruitment of college students for various job prospects, what qualities do students need to improve and enhance, especially in the post-epidemic era, and how the role of "cloud recruitment" are, all worth profound pondering.

Although the epidemic situation in China has been controlled, the spread of Covid-19 overseas has increased the uncertainty and instability of the global economy; the environment for resumption of work and for economic and social development is grim and complex, and the challenges are unprecedented (Ce.cn, 2020). Therefore, the epidemic will affect not only the students who are in the graduation year but also the junior students who are waiting to apply for admission to a graduation course. Unpredictability and uncertainty are now part and parcel of career development. Career adaptability, as the core ability of self-construction, is "the individual's readiness and coping resources when facing the uncertainty and complexity brought about by career transformation," (Savickas, 1997) and which have a significant potential impact on individual career development. Hirsch regards career adaptability as one of the key abilities for individual's career success (Hirschi, 2009). Some scholars are of the opinion that the development of career adaptability is the core process necessary for a successful career (Savickas, 2011). Moreover, many studies also show that career adaptability is closely related to health and mental well-being of individuals and thus they are positively correlated (Hirschi, 2009). Most of the people who have the ability to adapt and have, at some point, experienced adversity, are different from those that are vulnerable to some psychosocial factors. This study attempts to understand the coping strategies and the reaction of college students in the context of different career adaptability as a result of the sudden outbreak of the epidemic.

In the current scenario, there are several objective problems regarding the employment of university graduates in China, such as delayed graduation, employment difficulties, and settling down problems, which are all deeply concerning. However, under the epidemic impact, there are only a few empirical studies on the employment situation and employment psychology of Chinese 
college students, and most of them focus on the theoretical research of the function and practice of education under the Covid-19 outbreak (Yang, 2020; Liu, Yue et al., 2021).

For college graduates, spring is an important period for intensive defense of graduation thesis, implementation of employment units and preparation for studying abroad. However, the pace of employment has been disrupted by the sudden epidemic, which is undoubtedly an unexpected test. The major epidemic situation has a great impact on College Students' employment psychology, employment status and employment channels. The epidemic situation has changed the form of employment recruitment from offline to online, but the research on its effect and students' participation is lacking. At the same time, it is also worth paying attention to whether students' emotions, employment psychology, occupational adaptability and other internal factors will affect their use of the new employment recruitment platform. Based on this, this study focuses on the following issues:

Question 1. The effect on college students' employment as well as mental status during the major epidemic period. By focusing on career adaptability, which is the core competency factor, the study aims to explore its impact on the employment psychology and emotion of college students.

Question 2. The participation level of college students in various "cloud recruitment" employment information channels and the influence it has on the employment results.

\section{Research Methods}

\subsection{Research Object}

This study randomly selected junior and senior students from three universities as the research subjects; the students were dispersedly majoring in science, engineering, liberal arts, and arts. The participants had to complete the form and submit it between March 15 and March 25, 2020. The questionnaire was screened according to the response time and the repetition rate. A total of 1,300 questionnaires were collected, of which 1229 were valid; the effective rate thus was $94.54 \%$. The age of the subjects was $21.21 \pm 1.14$ years old. Among them, 548 students were juniors and 681students are seniors; 543 students were male and 686 students were female. A total of 510 students were majoring in science and engineering, and 719 students in liberal arts and arts. This research was approved by the Ethics Board of Nanjing Normal University and the questionnaires were completed voluntarily. All subjects voluntarily participated in the survey. Written informed consent from the [patients/participants OR patients/participants legal guardian/next of kin] was not required to participate in this study in accordance with the national legislation and the institutional requirements.

\subsection{Measuring Tools}

The questionnaire includes 69 questions in total and is divided into two parts: 
self-designed questionnaire and occupational adaptability scale. The coefficient of Cronbach's $\alpha$ in the whole scale is .922 .

The self-designed questionnaire involves five parts, which are as follows: the influence of the epidemic on employment/schooling, the influence of the epidemic on the mood, the influence of the epidemic on the employment mentality, the in-fluence of the employment mentality on the response to the epidemic situation, and the influence of the "cloud recruitment's" different employment information approaches. Some of the questions are category questions, while some are self-evaluation questions, which are scored on a scale of 5. Cronbach's a coefficient of the scale is .884 .

The career adaptability scale was revised by Hou Zhijin and others (Hou et al., 2012), and it includes four dimensions: career concern, career control, career curiosity, and career confidence. It includes 24 items in total and the responses are measured on a scale of 5. Cronbach's a coefficient of the scale is .966.

\subsection{Measuring Tools}

The study used SPSS statistical software to analyze the data.

\section{Research Results}

\subsection{The Situation of College Students' Employment/Academic Emotion Disturbed by Epidemic Situation}

The results of this study show that more than half of college students (69.3\%) have felt that from the time when the reopening of schools was postponed until the present scenario, the epidemic situation has caused significant emotional distress and has affected their employment and studies. In terms of the period of time the students were affected by this emotional distress, $62 \%$ suffered half a month or more, and only a small number of students suffered for a shorter period of less than 10 days. Also, based on the degree of emotional distress, the proportion of time required to relieve oneself from emotional distress is also different. In all, $40.7 \%$ of the participants were relieved from such emotional distress within 2 - 3 days, whereas $15.2 \%$ needed more than one month. As seen in Figure 1, as the impact of the epidemic on resumption of work/school aggravated, the duration of emotional distress in college students becomes longer. From the time the opening of schools was postponed until now, the longest duration of emotional distress is noted, which, in turn, needs longer time for college students to come to terms with emotional distress.

Generally speaking, the degree of emotional distress that senior students (graduation grade) go through is more than that of junior students. In terms of distress time and relief time, the degree of emotional distress that students majoring in liberal arts/arts go through is longer than that in students majoring in science/engineering. The duration of emotional distress in male students is longer than that noted in female students. However, in terms of emotional relief, although the time required for emotional relief by male students continues to 


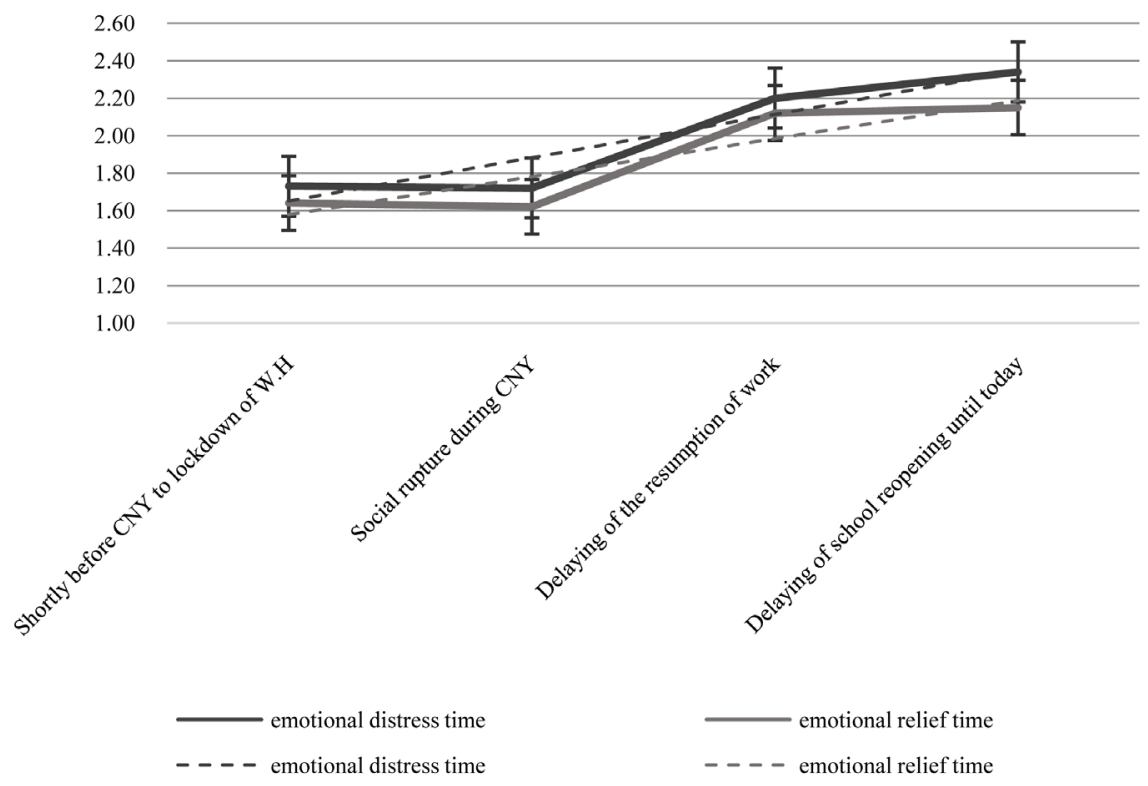

Figure 1. Comparison of emotional distress time and emotional relief time during different periods of the epidemic.

increase with the passage of the epidemic, it is still generally lower than that noted in female students. However, the duration of emotional distress in senior students forbidden to visit during the Chinese New Year (CNY) (social rupture during $\mathrm{CNY}$ ) until they find emotional relief has greatly decreased, and is lower than that noted in junior students. The duration of emotional distress in liberal arts/arts students, forbidden to visit during the CNY (social rupture during $\mathrm{CNY}$ ), until they got emotional relief has greatly reduced, and is lower than that noted in science/engineering students.

Overall, with the continuous impact of the epidemic, the emotional distress time in the group having low/medium career adaptability shows a straight line upward trend. The emotional distress time in the group having high career adaptability reaches the peak after there is delay in resumption of work after the CNY; however, over a period of time, the emotional distress time in the group with high career adaptability is alleviated and lower than that in the group with low/medium career adaptability (Figure 2). With the passage of time since the outbreak of the epidemic, in terms of emotional relief time, the group with low career adaptability requires significantly longer time than those in the group with medium/high career adaptability (Figure 3). The emotional relief time needed by those in the group with middle career adaptability increases with the ongoing of epidemic. However, the emotional relief time required by group with high career adaptability reaches the peak at the time when the resumption of work is delayed due to the epidemic situation (longer relief time than those in the medium career adaptability group, but shorter than those in the low career adaptability group), but as time passes by, the emotional relief time needed decreases, and it is shorter than that required by the medium/low career adaptability group. 


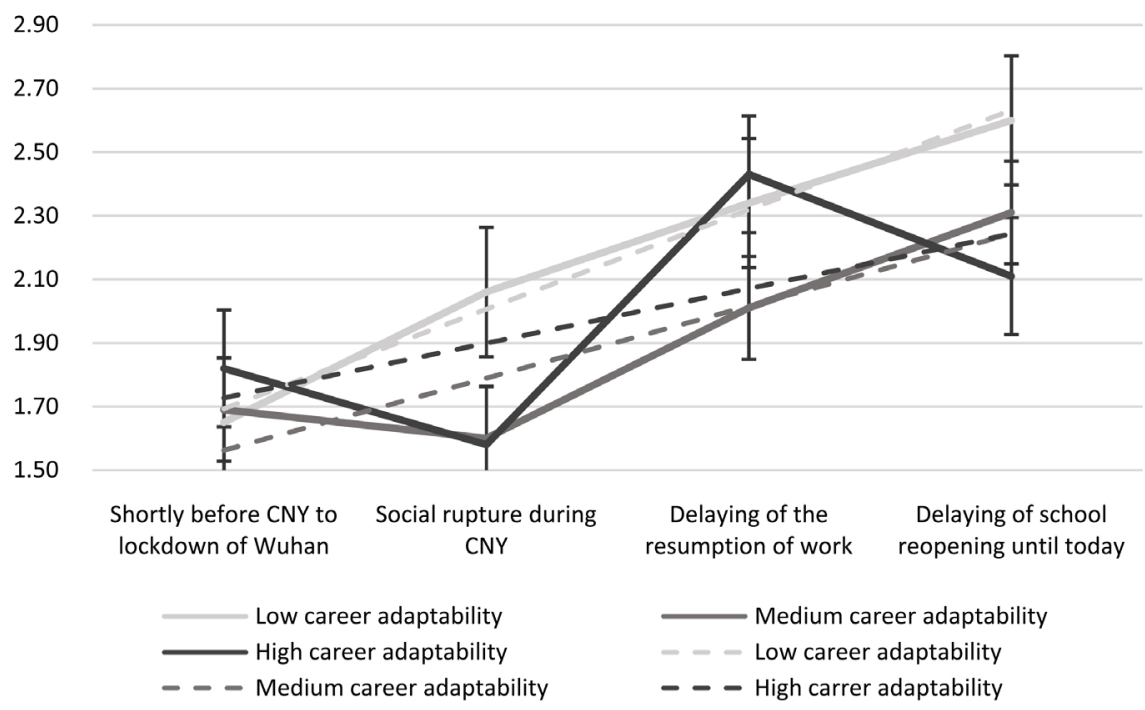

Figure 2. Comparison of emotional distress time of different career adaptability groups in different periods of epidemic.

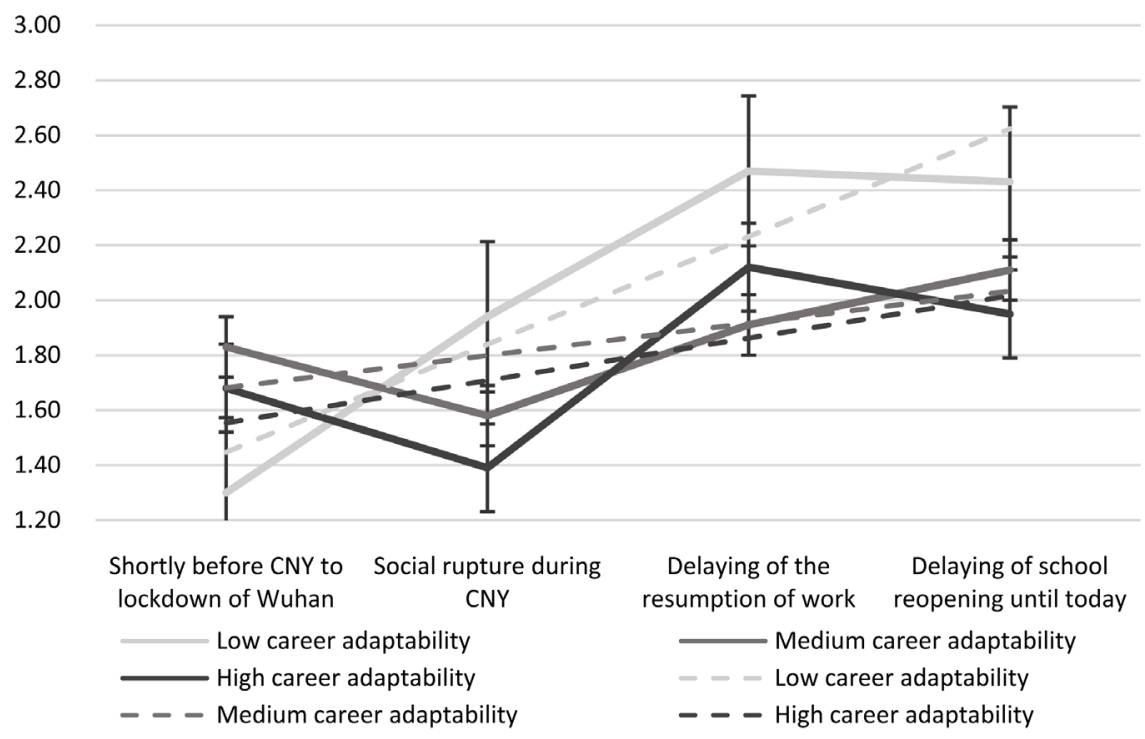

Figure 3. Comparison of emotional distress relief time of different career adaptability groups in different periods of epidemic.

\subsection{Units the Impact of Epidemic on Employment Mentality and the Influence of Employment Mentality on Employment Situation}

The impact of the epidemic on employment mindset may be positive or negative, but the positive effect accounts for more than 50\%. Among them, the epidemic has the most positive effect on being open and tolerant (77.5\%), on pursuing security and stability (76.2\%), and on helping others (73.4\%). The effect of the epidemic on employment status is divided almost equally, with half positive and the other half having negative feelings. Regardless of the positive or the negative effect the epidemic has on employment psychology, only taking into con- 
sideration the degree of influence, there are comparatively large groups (about $30 \%-40 \%$ ) who feel that the effect does exist (including a relative great impact and a very great impact).

In terms of grades, the influence of employment mentality in terms of pursuing security and stability on employment is significantly higher in senior students than in junior students $(t=-2.063, p<.05)$. The influence of a sense of professional mission on employment mentality is significantly higher in junior students than in senior students $(t=2.176, p<.05)$. Concerning gender, male students are more influenced by the employment mentality in terms of paying attention to economic remuneration and pursuing social prestige $(\mathrm{t}=3.463, p$ $<.01 ; \mathrm{t}=2.857, p<.01)$ than female students.

In general, in groups with high career adaptability (Table 1), the influence of different employment mentalities regarding the employment situation is significantly higher than that in groups with medium/low career adaptability $(p<.01$ or $p<.05$ ). However, the influence of mentality regarding the employment situation in terms of pursuing security and stability, staying open and tolerant, and helping others is higher in groups with medium career adaptability than in those with low career adaptability $(p<.05)$.

\subsection{The Influence of Different "Cloud Recruitment" Approaches on College Students' Employment Situation}

More than half of the participants (56.9\%) participated in various "cloud" recruitment activities. Among them, 25.7\% of the students think that these activities are beneficial $(10.4 \%$ of the students consider them greatly helpful, while $15.3 \%$ of the students consider them very helpful), more than half of the students (57.5\%) think the help is moderate, and the remaining (16.8\%) think that the help is small (6.2\% of them think the help is very small).In terms of participation in cloud-based recruitment, the school employment information platform attracts the most participation by students $(25.4 \%)$, followed by the third-party

Table 1. Variance test of the influence of various employment mentalities on the employment situation of groups with different career adaptability.

\begin{tabular}{|c|c|c|c|c|c|c|c|c|}
\hline & \multicolumn{2}{|c|}{$\begin{array}{l}\text { Low Career } \\
\text { Adaptability } \\
(\mathrm{N}=344)\end{array}$} & \multicolumn{2}{|c|}{$\begin{array}{l}\text { Medium Career } \\
\text { Adaptability } \\
\quad(\mathrm{N}=551)\end{array}$} & \multicolumn{2}{|c|}{$\begin{array}{l}\text { High Career } \\
\text { Adaptability } \\
(\mathrm{N}=334)\end{array}$} & \multirow[t]{2}{*}{$\mathrm{F}$} & \multirow[t]{2}{*}{ Multiple Comparison } \\
\hline & M & SD & M & SD & M & SD & & \\
\hline Pursuing Security and Stability & 3.47 & .89 & 3.64 & .75 & 3.80 & .95 & $12.80^{\star * *}$ & $1<2^{\star}, 1<3^{* \star *}, 2<3^{\star}$ \\
\hline Paying Attention to Economic Remuneration & 3.48 & .85 & 3.58 & .72 & 3.71 & .97 & $6.13^{* * *}$ & $1<3^{* *}$ \\
\hline Pursuing Social Prestige & 3.16 & .82 & 3.24 & .69 & 3.49 & 1.05 & $14.50^{* * *}$ & $1,2<3^{* * *}$ \\
\hline Sense of Professional Mission & 3.31 & .89 & 3.45 & .72 & 3.78 & .94 & $29.05^{\star * *}$ & $1,2<3^{\star * *}$ \\
\hline Staying Open and Tolerant & 3.35 & .83 & 3.57 & .76 & 3.90 & .95 & $37.71^{\star * *}$ & $1<2,3^{* * *}, 2<3^{* * *}$ \\
\hline Helping Others & 3.31 & .84 & 3.44 & .73 & 3.76 & .99 & $26.63^{* * *}$ & $1<2^{*}, 1,2<3^{* * *}$ \\
\hline
\end{tabular}

Note: ${ }^{* * *} p<.001,{ }^{* *} p<.01,{ }^{*} p<.05$ 
recruitment platform (19.8\%), target employer information platform (13.3\%), teacher/employment counselor (12.7\%), governmental employment information platform (10\%), family (9.7\%), and friends (9\%). However, as far as help for college students is concerned (Figure 4), the target employer information platform plays the most important role, followed by the third-party recruitment platform, the help of family, school employment information platform, teachers and employment counselors, and friends.

Overall, compared to the senior students, the junior students think that all kinds of "cloud recruitment" approaches are helpful, especially approaches such as school (websites, official employment accounts) employment information platforms $(\mathrm{t}=1.975, p<.05)$, governmental employment information platforms $(\mathrm{t}=4.097, p<.01)$, target employer information platforms $(\mathrm{t}=3.221, p<.05)$, and friends $(\mathrm{t}=2.332, p<.05)$. Compared to students majoring in liberal arts/arts, students majoring in science/engineering are more likely think that various employment approaches are more helpful to their employment, especially approaches such as school (website, official employment accounts) employment information platforms $(\mathrm{t}=4.831, p<.01)$, governmental employment information platforms $(\mathrm{t}=2.84, p<.05)$, target employer information platforms $(\mathrm{t}=2.012, p<.05)$, third-party recruitment platforms $(\mathrm{t}=2.358, p<.05)$, and teachers/employment counselors $(\mathrm{t}=3.526, p<.01)$.

Compared with the group having low/medium career adaptability, the group with high career adaptability reckons that various "cloud recruitment" approaches are significantly helpful $(p<.01$ or $p<.05)$ (Table 2$)$. Compared with the group having low career adaptability, the group with medium career adaptability more likely reckons that various "cloud recruitment" approaches are significantly helpful $(p<.01$ or $p<.05)$.

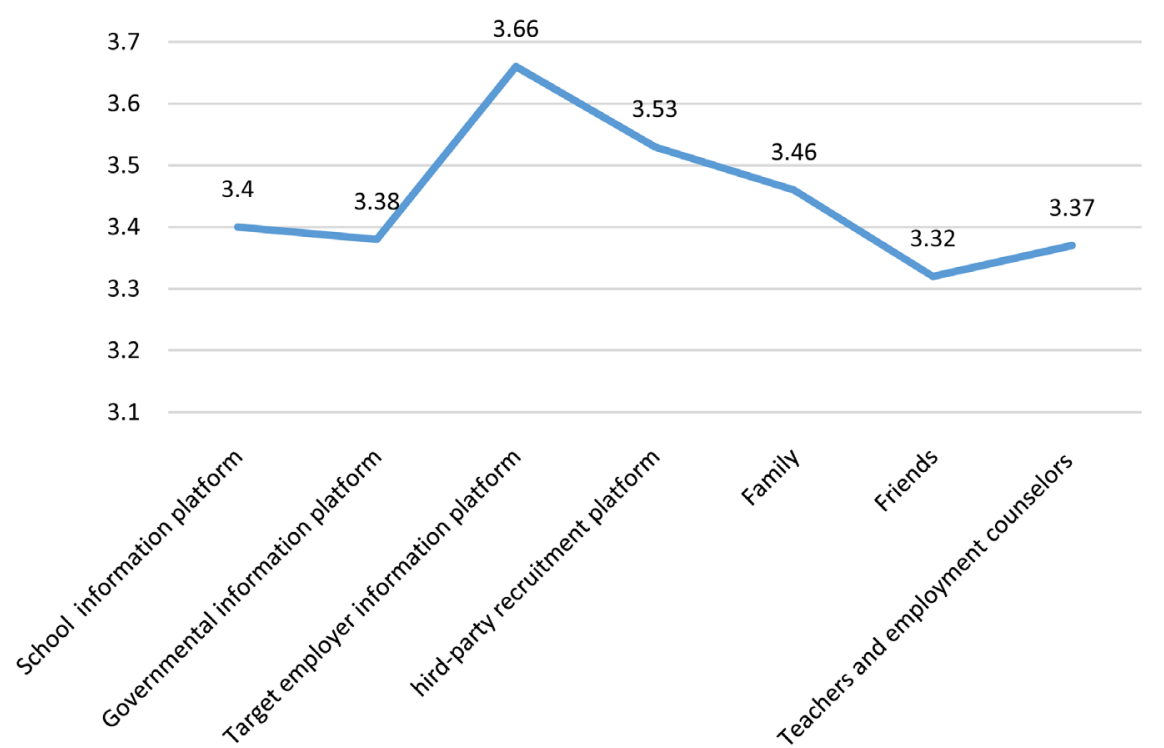

Figure 4. Comparison of the assistance of different "cloud recruitment" approaches for college students' employment situation. 
Table 2. Variance test of the employment effect of various employment approaches on groups with different career adaptability.

\begin{tabular}{|c|c|c|c|c|c|c|c|c|}
\hline & \multicolumn{2}{|c|}{$\begin{array}{l}\text { Low Career } \\
\text { Adaptability } \\
(\mathrm{N}=344)\end{array}$} & \multicolumn{2}{|c|}{$\begin{array}{c}\text { Medium Career } \\
\text { Adaptability } \\
(\mathrm{N}=551)\end{array}$} & \multicolumn{2}{|c|}{$\begin{array}{l}\text { High Career } \\
\text { Adaptability } \\
(\mathrm{N}=334)\end{array}$} & \multirow[t]{2}{*}{$\mathrm{F}$} & \multirow[t]{2}{*}{ Multiple Comparison } \\
\hline & M & $\mathrm{SD}$ & $\mathrm{M}$ & $\mathrm{SD}$ & M & $\mathrm{SD}$ & & \\
\hline School Employment Information Platforms & 3.22 & .87 & 3.44 & .83 & 3.54 & .98 & $11.75^{\star * *}$ & $1<2^{\star *}, 1<3^{* * *}$ \\
\hline Governmental Employment Information Platforms & 3.18 & .84 & 3.40 & .81 & 3.54 & 1.00 & $14.24^{* * *}$ & $1<2,3^{* * *}$ \\
\hline Target Employer Employment Information & 3.37 & .86 & 3.69 & .80 & 3.93 & .95 & $35.55^{\star * *}$ & $1<2,3^{\star * *}, 2<3^{\star * *}$ \\
\hline Third-party Recruitment Information Platforms & 3.35 & .80 & 3.52 & .77 & 3.75 & .89 & $21.36^{* * *}$ & $1<2^{\star *}, 1,2<3^{\star * *}$ \\
\hline Family & 3.27 & .91 & 3.48 & .83 & 3.61 & 1.03 & $12.19^{* * *}$ & $1<2^{\star *}, 1<3^{\star *}$ \\
\hline Friends & 3.16 & .84 & 3.32 & .79 & 3.48 & .97 & $11.44^{* * *}$ & $1<2^{* *}, 1<3^{* * *}, 2<3^{*}$ \\
\hline
\end{tabular}

Note: ${ }^{* *} p<.001,{ }^{* *} p<.01,{ }^{*} p<.05$.

\section{Analysis and Discussion}

\subsection{Emotional Distress to College Students' Employment/Study Caused by the Delayed Reopening of Schools}

More than half $(69.3 \%)$ of the sample of college students have obviously experienced the adverse impact of the epidemic that caused a delay in the reopening of schools. It can be seen from Figure 1 that with the impact of the epidemic on joining work and resuming schools, the longer the duration of emotional distress, the longer is the time required to alleviate the distress, which is consistent with the research results of some researchers. For example, on the basis of their research, Li Shaowen and others found that adolescents had generally different degrees of depression and anxiety during the epidemic period. It has also been noticed that the degree of emotional distress gradually flattens over time. In particular, the final-year students are faced with a series of issues, such as defending their research paper, job-hunting, the postgraduate entrance examination, and preparation for further education abroad. The sudden epidemic is not only disruptive but also holds in suspense many matters related to graduating, such as the postponement of defense of the graduation paper and the postgraduate entrance examination, the increased risk of unemployment, and the suspension of overseas visa, all of which have immensely impacted the mental well-being of graduates. Therefore, under the epidemic situation of Covid-19, compared with the junior students, the degree of distress senior students experience becomes deeper with each passing day.

Although the degree of emotional distress in students majoring in science and engineering increases with the progression of the ongoing of epidemic, it is generally lower than that of students majoring in liberal arts and arts. Based on the trend of past years, the best time for employment is from October to December and from March to April. However, participants exhibit different situations in different majors. For students majoring in science and engineering, the peak season for finding a job is mostly in autumn, while for those majoring in liberal 
arts/arts majors are mostly in spring semester. For students of liberal arts/arts majors, the employment contracts are mostly concentrated in the spring semester. So the impact of the epidemic on their employment situation is predictable. The impact of the epidemic on gender is also different. Male students experience longer emotional distress than female students, but the time needed to obtain relief from stress is generally shorter. Although male students experience stronger emotional ups and downs, they are more capable to cope with it. Female students are more emotionally stable, but they tend to be more sensitive, thus making them less capable to obtain relief from stress.

\subsection{The Coexistence of "Risks" and "Opportunities" in the Influence of the Epidemic on Employment Mentalities}

The impact of the epidemic on the employment mentality can be both positive and negative, but the negative effect is predominant. With regard to the influence of the epidemic on employment confidence, the positive and negative effects are almost equal ( $52 \%$ vs. $48 \%$ ), with the negative effects slightly outnumbered. In fact, there is a difference between this proportion of employment confidence acquired under epidemic situation and that acquired under normal circumstances; for example, according to a survey by some scholars, $90 \%$ of the students under normal circumstance) were confident of successful employment in the future. In all, $77.5 \%$ of the student participants think that the epidemic situation has a positive effect on their open and inclusive employment mentality, $76.2 \%$ of the students consider the epidemic as a positive effect on their employment mentality of pursuing security and stability, and $73.4 \%$ of the students believe that the epidemic has a positive effect on their employment mentality of helping others. Such positive effects on their employment mentality are "opportunity" and the "self-development" brought by the epidemic situation.

Under the current epidemic crisis, the influence of employment mentality on employment becomes obvious. Compared to junior students, senior students experience profound influence on employment which is brought about by the pursuing of security and stability. At present, the economic and social challenges are still prominent, including both short-term difficulties that are caused by the epidemic situation, and the long-term and structural problems that bring risks and uncertainties to employment. As the primary aim of graduates, the importance of employment is self-evident. It is also understandable that compared to junior students, senior students experience a stronger influence on employment as a result of pursuing safety and stability. In addition, compared to female students, male students experience significantly more influence on employment as a result of mentalities such as paying attention to economic remuneration and pursuing social prestige. Sociological researches show that income, power, education level, and the nature of employment are the main factors that are considered in terms of professional prestige in China. Students usually yearn for a career with high income and a certain level of power. In the society we live in, we find that the society claims higher requirement for men, expecting that men can 
pursue success and can gain both fame and wealth. On the contrary, the society has lower occupational expectation from women, thus making them more eager to pursue psychological self rather than considering the social prestige, the economic reward, and so forth (Gottfredson, 1981).

\subsection{Different “Cloud Recruitment" Approaches Vary in Levels of Participation and Roles}

It is clear that, firstly, the "cloud recruitment" played an important role in the employment of students during the epidemic period, which is an innovative and guaranteed methodology. Secondly, the third-party "cloud recruitment" platform is cost-effective, user-friendly, and also useful for employment. Thirdly, the "cloud recruitment" platform in colleges and universities enjoy the highest degree of participation from students; however, its role needs to be strengthened, which can be done by putting forward new requirements for the construction of employment in-formation platform in colleges and universities in the future. As an information platform often used by students, how to improve its value is a new topic requiring greater involvement of university employment departments.

Moreover, based on the survey, it was found that although the target employer employment information platform is not the most used, it is considered to be the most helpful and is way ahead of other employment information platforms and channels in terms of the usefulness (Figure 4). Determining career goals is an important step in career decision-making, and person-post matching is considered to be an important indicator to evaluate the effectiveness of career decision-making (Singh \& Greenhaus, 2004). A wise job selection is that which achieves the best match between oneself and the job (Edwards, 1991). In some studies, person-post matching has been directly used to measure the effectiveness of career decision-making (Singh \& Greenhaus, 2004). This view point thus supports the fact that the target employer employment information platform is of great help. The clearer the goal is, the clearer the future is, and the more likely it is to make a satisfactory decision: this coincides with the view of some researchers. They find that the decision satisfaction level in people with high future time perspective is more than that in people with low future time perspective, that is, people's vision of the future will have a profound impact on their own current state of mind and action.

\subsection{Unexpected Situations Require More Career Adaptability}

As seen from Table 1, career adaptability is significantly positively correlated with the influence of the epidemic on the employment mentality, and negatively correlated with the degree of emotional distress. With the ongoing epidemic crisis, the group with high career adaptability experiences lower degree of emotional (distress time and relief time) compared to the group with medium/low career adaptability. Although the degree of emotional distress in the group with high career adaptability group reaches the peak before the resumption of work, it still exceeds that in the group with medium/low career adaptability; however, 
with the passage of time, their emotional distress level is greatly alleviated. On the other hand, the degree of emotional distress experienced by the group with low career adaptability continues to increase with the continuous impact of the epidemic, and it is far higher than that of the group with medium/high career adaptability. This proves once again that career adaptability has a direct effect on individual's physical and mental health: positive career orientation can reduce negative emotions. It also shows that when one is confronted with unexpected events that have a sudden impact on the career and employment, regardless of the long duration, the group with high career adaptability can quickly adjust their mentality, which agrees with research by Tolentino et al. (2014) and others who claim that the career adaptability is positively correlated with occupational optimism.

Moreover, the employment of the group with high career adaptability is mostly influenced by their various employment mentalities. The employment mentalities of the group with high career adaptability such as pursuing security and stability, staying open and tolerant, and helping others have a significantly higher effect on employment than that of the group with low/medium career adaptability. Career adaptability is when one is "willing to deal with predictable tasks in work, and unforeseen changes in the profession and working conditions." Because of the epidemic, small and medium-sized enterprises have been affected to varying degrees, because of which people's employment mentality has also changed. From the research results, we notice that the epidemic has a positive effect on the employment mentalities like pursuing security and stability, staying open and tolerant, and helping others which all affect individual employment choice. Apparently the group with high career adaptability is seen to have significantly experienced more positive effects. It reflects that the career adaptability, as a psychological resource, is the ability of individuals to interact with the environment in order to adapt to career changes, which changes due to the interaction with the environment, which is a dynamic construction process. People with high career adaptability can digest and process discomfort more quickly than those with medium/low career adaptability (Johnston et al., 2013) and can even predict the high level people-post matching perception in future jobs (Guan et al. 2013). Compared to the people with medium/low career adaptability, the people with high career adaptability think that various cloud recruitment approaches pose significantly more help in terms of employment. The adaptive behavior and the results are the output of the aftereffect adaptation. Thus, individuals with strong adaptability will show more positive behaviors to actively participate in career exploration (Urbanaviciute et al. 2014), cherish every opportunity for improvement, and will look on more positively at external aids.

\section{Suggestions and Strategies}

Although epidemic crisis has caused certain emotional distress in terms of the employment and the study of college students, its effect can also be positive. This 
influence is beyond the emotional distress and has a reshaping effect on the employment mentality of various dimensions, which makes young students aware of the parts ignored or condemned in the past. Therefore, the epidemic crisis is not only a "battlefield" for the employment but also a "classroom" for the growth of young students. We can see the positive role of epidemic in promoting students' employment mentalities, such as pursuing security and stability, helping others, and staying open and tolerant, which all provide a favorable basis for the value re-construction of employment. However, this is only an instinctive response to objective factors rather than the inevitable shaping process of subjective consciousness. In this process, the school employment work should not only focus on the single dimensional goal of "employment" but also help students to jump out of "small situation" and "small time," face the grand "grand environment" and the "grand era," guide students to establish the "micro employment concept" to deal with the current situation, and lead students to establish a "grand career outlook" and a "grand life outlook" focusing on the future. Thus, the time for reshaping employment values, the content framework of employment values, and the method carrier of employment values are worth researching. Neither early nor late intervention can achieve the desired effect. In terms of content, the following three themes are indispensable: the guidance of ideal and belief, the social responsibility education, and the career mission construction. Therefore, the shaping of employment outlook for future generation should focus not only on the cultivation of the individual will of "profession" and "employment" but also on the guidance of collective values of "industries" and the "nationality."

The employment condition under the epidemic crisis has changed from the "single soldier operation" and employment entity of the past. Instead, it has broken the border barriers and turned to full staff cooperation and online employment. Based on the number of participants in the study, the participation in the online recruitment platforms is lower than expected, accounting for only $56.9 \%$. Only $25.7 \%$ of the students think that the help regarding getting employment is commendable. Objectively speaking, by inspecting some previous survey data, the participation and the assistance of online recruitment channels have improved, but they require still more upgrading. However, according to online comments, many students clock in, but they generally keep silent and rarely participate by asking questions; thus, the effect of such online platforms needs further discussion. Additionally, how to improve the participation and the reflection of students during these sessions and give full play to the role of employment cloud platform is worth discussing. Besides the fact that employment cloud platform is the result of sudden emergencies and its effect has not yet been fully revealed, the main reason is that many young students do not have a strong sense of career goal, and the "unambitious" group accounts for a high proportion. The research results show that although the target employer employment platforms are the most helpful, they are not the most frequently used. This is because many students do not know what their job objectives are and what they 
want to do. Thus, confusion has become the mantra and many take refuge in it. The emerging "employment Internet field" is the means and the carrier to deal with the outbreak of the epidemic crisis, and the fundamental problem to be solved is to help establish career goals for students, because clear goals lead to clear paths.

The academic community is increasingly aware that "the flow of knowledge age" is increasingly becoming borderless, changeable, and value-conflicted. The epidemic crisis brings up natural and non-human factors to the "uncertain reality." This objective changes, which is not based on human will, brings about the "uncertain future," which proves that the adaptation is the key to career development whose eternal theme is about "change" and "change-coping." As a personal ability to respond appropriately to the "uncertain reality," the adaptation is a dynamic construction process in which the individual actively pursues self-adaptation to the external environment which is the internal scale of individual cognitive professional role and the external scale of the spatial and temporal structure of professional practice, as well as the active construction process of internal and external interaction. In this study, the adaptability, as an ability or a stable psychological contract in dealing with emergencies, has proved its advantage, such as low emotional distress and emotion relief manifested in the high career adaptability groups in the epidemic crisis, or the timely adjustment of self-employment mentality to cope with the employment impact of the epidemic crisis. As university employees, the work of employment requires provision not only of information to help students get employed but also of education to aid students to develop in the future (Perera \& McIlveen, 2014). However, in terms of the essence of individual development, it is an integral part of the interaction and construction of environment and cannot be separated. Therefore, adaptation is not a passive bearing but is an active response, not only facing the present but also continuing into the future (Skorikov \& Vondracek, 2007). We can discern some clues from the logic of career adaptability regarding what is individual "change" and "change-coping." The psychological structure of attention independence-curiosity-confidence and the spiral rise of interaction with environment constitute the personal "change" and "change-coping." In this process, passivity turns into initiative, and initiative turns into psychological capital, which finally achieves "balance" and "smoothness." As a mechanism to promote adaptation and development of college career education, "change" and "unchanged", "change" and "change-coping" are also worth pondering. Adaptation, which is applicable to the career education mechanism in colleges and universities, is the response of career education mechanism to "changing external environment" and "different majors with specialties/employment individual." What is "unchanged" is the essence and purpose of education, while what is "changed" is the method and response.

\section{Acknowledgements}

The authors would like to thank the reviewers for their valuable comments on 
the manuscript.

\section{Funding}

This research was funded by Jiangsu Province University's Advantageous Discipline Construction Project, grant number "PAPD". The study was also supported by employment and Entrepreneurship of college graduates in Jiangsu Province, China. Topic is the Research on the design of career education flipped classroom to promote students' future time insight, (Grant No. JCKT-2019508).

\section{Conflicts of Interest}

The authors declare no conflicts of interest regarding the publication of this paper.

\section{References}

Ce.cn. (2020). Why Should Firm the Chinese Confidence That Wins?

Edwards, J. R. (1991). Person-Job Fit: A Conceptual Integration, Literature Review and Methodological Critique. In: Cooper, C.L. and Robertson, I.T., Eds., International Review of Industrial Organizational Psychology (Vol. 6, pp. 283-357). Wiley.

Gottfredson, L. S. (1981). Circumscription and Compromise: A Developmental Theory of Occupational Aspirations. Journal of Counseling Psychology, 28, 545-579. https://doi.org/10.1037/0022-0167.28.6.545

Grillon, C., Baas J. P., Lissek, S., Smith, K., \& Milstein, J. (2004). Anxious Responses to Predictable and Unpredictable Aversive Events. Unpredictable Aversive Events. Behavior Neuroscience, 118, 916-924. https://doi.org/10.1037/0735-7044.118.5.916

Guan, Y., Deng, H., Sun, J., Wang, Y., Cai, Z., Ye, L. et al. (2013). Career Adaptability, Job Search Self-Efficacy and Outcomes: A Three-Wave Investigation among Chinese University Graduates. Journal of Vocational Behavior, 83, 561-570.

https://doi.org/10.1016/j.jvb.2013.09.003

Hirschi, A. (2009). Career Adaptability Development in Adolescence: Multiple Predictors and Effect on Sense of Power and Life Satisfaction. Journal of Vocational Behavior, 74, 145-155. https://doi.org/10.1016/j.jvb.2009.01.002

Hou, Z. J., Leung, S. A., Li, X., \& Xu, H. (2012). Career Adapt-Abilities Scale-China Form: Construction and Initial Validation. Journal of Vocational Behavior, 80, 686-691. https://doi.org/10.1016/j.jvb.2012.01.006

Johnston, C. S., Luciano, E. C., Maggiori, C., Ruch, W., \& Rossier, J. (2013). Validation of the German Version of the Career Adapt-Abilities Scale and Its Relation to Orientations to Happiness and Work Stress. Journal of Vocational Behavior, 83, 295-304. https://doi.org/10.1016/j.jvb.2013.06.002

Liu, Y., Yue, S., Hu, X., Zhu, J., \& Wu, Y. (2021). Associations between Feelings/Behaviors during COVID-19 Pandemic Lockdown and Depression/Anxiety after Lockdown in a Sample of Chinese Children and Adolescents. Journal of Affective Disorders, 284, 98-103. https://doi.org/10.1016/j.jad.2021.02.001

Perera, H. N., \& McIlveen, P. (2014). The Role of Optimism and Engagement Coping in College Adaptation: A Career Construction Model. Journal of Vocational Behavior, 84, 395-404. https://doi.org/10.1016/j.jvb.2014.03.002

Savickas, M. L. (1997). Adolescent Career Development in Social Context. Career Devel- 
opment Quarterly, 45, 303-304. https://doi.org/10.1002/j.2161-0045.1997.tb00535.x

Savickas, M. L. (2011). Career Adaptability: An Integrative Construct for Life-Span, Life-Space Theory. Career Development Quarterly, 45, 247-259.

https://doi.org/10.1002/j.2161-0045.1997.tb00469.x

Singh, R., \& Greenhaus, J. H. (2004). The Relation between Career Decision-Making Strategies and Person-Job Fit: A Study of Job Changers. Journal of Vocational Behavior, 64, 198-221. https://doi.org/10.1016/S0001-8791(03)00034-4

Skorikov, V., \& Vondracek, F. W. (2007). Positive Career Orientation as an Inhibitor of Adolescent Problem Behavior. Journal of Adolescence, 30, 131-146. https://doi.org/10.1016/j.adolescence.2006.02.004

Tolentino, L. R., Garcia, P. R. J. M., Lu, V. N., Restubog, S. L. D., Bordia, P., \& Plewa, C. (2014). Career Adaptation: The Relation of Adaptability to Goal Orientation, Proactive Personality, and Career Optimism. Journal of Vocational Behavior, 84, 39-48. https://doi.org/10.1016/j.jvb.2013.11.004

Urbanaviciute I., Kairys, A., Pociute, B., \& Liniauskaite, A. (2014). Career Adaptability in Lithuania: A Test of Psychometric Properties and a Theoretical Model. Journal of Vocational Behavior, 85, 433-442. https://doi.org/10.1016/j.jvb.2014.09.005

Yang, T. (2020). Ways and Methods to Promote the Mature Growth of College Students in Response to Major Epidemic Diseases. The Theoretical Construction, 36, 15-17.

Zhang, X., Hou, Z., Zhu, J., \& Wang, D. (2018). Influence of Fuzzy Tolerance on Career Decision-Making Difficulty: The Mediating Effect of Career Adaptability and Anxiety. Chinese Journal of Clinical Psychology, 2, 347-352. 